\title{
Simulation of two-phase flow through ducts with discontinuous cross-section
}

\author{
S. Brown*, S. Martynov, H. Mahgerefteh \\ Department of Chemical Engineering, University College London, London WC1E 7JE
}

\begin{abstract}
The development of an AUSM+-up based scheme for simulating transient single or two-phase flows through ducts with discontinuous or abrupt changes in area is presented. The non-conservative terms in the governing equations arising from the variation in the duct area are discretised to maintain exactly states at rest. An additional scaling of the pressure based dissipation is added to ensure numerical stability across the area change. The extensive application of the scheme to ideal gas and two-phase $\mathrm{CO}_{2}$ based on the Homogeneous Equilibrium Model (HEM) for both shock tube and other transient flow problems indicate the scheme's capability to resolve such problems accurately and robustly.
\end{abstract}

Keywords: Multi-phase flow, Shock tube, Non-conservative system

\section{Introduction}

In many engineering applications, the modelling of transient compressible two-phase or flashing flows in ducts with discontinuous cross-sections is essential given that this information determines the flow efficiency and heat transfer processes. Examples include cavitation in automotive fuel injection systems (Martynov et al., 2006), liquid flashing and gas condensation during operational or emergency venting of hydrocarbon pipelines (Ettouney et al., 2012), flash boiling of water during loss-of-coolant accidents in nuclear reactors (Nigmatulin and Soplenkov, 1994) and liquid boiling and expansion in refrigeration systems and heat pumps (Simões-Moreira and Bullard, 2003). Importantly, many of these systems must be treated as one-dimensional with abrupt area changes

\footnotetext{
${ }^{*}$ Corresponding author

Email address: solomon.browneucl.ac.uk (S. Brown )
} 
where the flow undergoes rapid expansion or contraction (Blinkov et al., 1993) often leading to a phase change.

In recent years, for the simple case of an ideal gas, a number of authors have studied such problems numerically, leading to the development of a number of schemes designed to be 'well-balanced' (Bouchut, 2004) in order to capture the correct wave behaviour at the discontinuity in duct area (Helluy et al., 2012; Clain and Rochette, 2009). For the flow of an ideal gas through porous media, Rochette et al. (2005), for example, constructed a simple VFRoe based well-balanced scheme. Kröner and Thanh (2006), on the other hand, presented a well-balanced scheme based on the Lax-Friedrichs flux which was later extended by Thanh and Kroner (2012) who added a nonlinear equation to ensure calculation of the correct wave configurations. This method was shown to perform reasonably well even for complex resonant problems. Rochette et al. (2012) studied the scheme presented by Clain and Rochette (2009) for a number of transient flow problems and showed that it was in general capable of computing the correct wave configuration.

The non-conservative term present in the governing equations is analogous to that in the onedimensional multi-fluid model (Stewart and Wendroff, 1984) and is a result of the averaging procedures used in their respective derivations (Ishii and Hibiki, 2006; Abgrall and Saurel, 2003). Given their importance for the modelling of two-phase flows, a great deal of research has focussed on the numerical solution of the multi-fluid equations (see for example Cortes, 2002; Yeom and Chang, 2013; Saurel et al., 2008). A scheme which has received attention in this respect is the AUSM+-up of Liou (2006), first applied to the two-fluid model by Paillere et al. (2003). Importantly, Liou et al. (2008) presented a further adaptation of this scheme by discretising the nonconservative pressure terms in such a manner as to maintain the well-balanced property, but only at rest. This property was shown to alleviate unphysical oscillations previously observed in the numerical solutions. The similar structure of the multi-fluid equations and those governing flow in ducts of varying cross-sectional area implies the applicability of an extension of this methodology to this latter problem.

For the simulation of flows with phase transitions the Homogeneous Equilibrium Model (HEM) represents the simplest two-phase model capable of simulating phase transitions and has been used extensively for the simulation of, for example, cavitation (see for example Goncalvès and Patella, 
2009, 2010). A benefit of this model is that its structure and mathematical character are almost identical to those of a single-phase fluid, hence the application of schemes developed for such fluids are readily extended to the HEM (Edwards et al., 2000).

Various methods have been devised for the HEM and its extension, the homogeneous relaxation model, (Faucher et al., 2000; Edwards et al., 2000) for simulating flows undergoing phase transitions in ducts of varying cross-sectional areas.

However, despite its obvious practical relevance, no literature incorporating discontinuous or abrupt changes in their cross-sectional area is available. This is primarily due to the difficulties associated with dealing with the loss of hyperbolicity of the governing equations at the cross-sectional area discontinuity. Such a loss makes their numerical solution complicated (Rochette et al., 2005) as it requires handling non-classical wave configurations (known as resonance) and may suffer from non-uniqueness (see for example Andrianov and Warnecke, 2004; Han et al., 2012).

The aim of this paper is, for the first time, to develop a methodology for resolving transient twophase flows in ducts with discontinuous changes in cross-sectional area.

The work proceeds as follows: Section 2 presents the mathematical model for one-dimensional flow in ducts of variable area. Section 3 describes the numerical solution technique based on the adaptation of the AUSM+-up scheme (Liou et al., 2008) paying particular attention to the discretisation of the non-conservative terms given the importance of resolving this for the accurate solution of the equations.

Section 4 presents an analysis of the AUSM+-up scheme's performance based on its application to a number of test problems involving flow through ducts with discontinuous areas for which either an analytical or reference solution is available.

Section 5 presents the application of the scheme to a number of transient two-phase problems in order to demonstrate its capability in capturing the pertinent flow phenomena including phase change and shock wave propagation. $\mathrm{CO}_{2}$ is chosen as the working fluid given its extensive use as a refrigerant and its role in Carbon Capture and Sequestration (CCS), in particular during its pipeline transportation where flow through discontinuities is often encountered. Conclusions are drawn in Section 6. 


\section{Model formulation}

\subsection{Fluid dynamics}

The governing equations for one-dimensional single-fluid flow in a rigid duct of variable crosssectional area are based on the Euler equations with an additional non-conservative term, (Faucher et al., 2000):

$$
\frac{\partial \mathbf{U}}{\partial t}+\frac{\partial \mathbf{F}(\mathbf{U})}{\partial x}=\mathbf{S}
$$

where

$$
\mathbf{U}=\left(\begin{array}{c}
\rho A \\
\rho u A \\
\rho E A \\
A
\end{array}\right), \mathbf{F}(\mathbf{U})=\left(\begin{array}{c}
\rho u A \\
\rho u^{2} A+A P \\
\rho u H A \\
0
\end{array}\right), \mathbf{S}=\left(\begin{array}{c}
0 \\
P \frac{\partial A}{\partial x} \\
0 \\
0
\end{array}\right)
$$

$u$ and $\rho$ on the other hand are the fluid velocity and density respectively. $P$ is the system pressure and $A$ is the duct area. $E$ and $H$ represent the specific total mixture energy and total enthalpy respectively defined as:

$$
\begin{aligned}
& E=e+\frac{1}{2} u^{2} \\
& H=E+\frac{P}{\rho}
\end{aligned}
$$

where $e$ is the specific internal energy.

Importantly, the system of equations (1) is non-strictly hyperbolic (Thanh and Kroner, 2012). In particular as the eigenvalues of the Jacobian of the flux function $\mathbf{F}(\mathbf{U})$ are given by $\lambda_{1}=u-a$, $\lambda_{2}=u, \lambda_{3}=u+a$ and $\lambda_{4}=0$, where $a$ is the fluid speed of sound. Therefore, in cases where $\lambda_{1}=\lambda_{4}=0$ or $\lambda_{3}=\lambda_{4}=0$ the system becomes non-hyperbolic and the solutions are non-classical, and are referred to as resonant. 


\subsection{Homogeneous Equilibrium Model}

For the purposes of modelling flashing liquid flows, the Homogeneous Equilibrium Model (HEM) is utilised, in which the liquid and vapour phases are assumed to be in both thermal and mechanical equilibrium, i.e. they share the same temperature and velocity. Accordingly, the mixture density and internal energy are respectively defined as:

$$
\begin{gathered}
\rho=\sum_{k} \alpha_{k} \rho_{k} \\
\rho e=\sum_{k} \alpha_{k} e_{k} \rho_{k}
\end{gathered}
$$

where $\alpha_{k}$ is the volume fraction of phase $k$ which must satisfy:

$$
\sum_{k} \alpha_{k}=1
$$

In the present work the thermodynamic properties for both the liquid and vapour phases are determined using the Peng-Robinson (PR) equation of state (Peng and Robinson, 1976):

$$
P=\frac{R T}{v-b}-\frac{c \delta}{v^{2}+2 b v-b^{2}}
$$

Where, $T, v$ and $R$ are the temperature, specific volume and the gas constant respectively. $c, b$ and $\delta$ are respectively given by:

$$
\begin{gathered}
c=0.45724 \frac{\left(R T_{c}\right)^{2}}{P_{c}} \\
b=0.07780 \frac{R T_{c}}{P_{c}} \\
\delta=\left[1+\left(0.37464+1.54226 \omega-0.26992 \omega^{2}\right)\left(1-\sqrt{\frac{T}{T_{c}}}\right)\right]^{2} .
\end{gathered}
$$

Where, $P_{c}, T_{c}$ and $\omega$ are respectively the critical pressure and temperature and the acentric factor, 
which for $\mathrm{CO}_{2}$ are given by (Poling et al., 2001):

$$
P_{c}=72.44016 \text { bara, } T_{c}=304.35 \mathrm{~K} \text { and } \omega=0.2236
$$

From equation (7), the internal energy may be calculated using the identity (Poling et al., 2001):

$$
e-e_{i g}=\int_{\infty}^{v}\left[T\left(\frac{\partial P}{\partial T}\right)_{v}-P\right] d v
$$

where the subscript, $i g$ refers to ideal gas. In the case where the fluid is single phase, either vapour or liquid, equations (8) and (7) are solved iteratively in order to calculate the values of $P$ and $T$. Where the fluid is a two-phase mixture equations (4), and (5), utilising equations (8) and (7), are solved by iterating the mixture pressure, $P$ giving the saturated temperature $T$, calculated from equation (7) (see example Hammer et al., 2013, for a full description).

The speed of sound for each individual phase $k$ is calculated using:

$$
a_{k}^{2}=\frac{\gamma_{k}}{\kappa_{k} \rho_{k}}
$$

where $\gamma$ is the ratio of specific heats and $\kappa$ is the isothermal coefficient of volumetric expansion (Picard and Bishnoi, 1987). For two-phase mixtures the speed of sound is calculated using the following expression:

$$
\frac{1}{\rho a^{2}}=\sum_{k} \frac{\alpha_{k}}{\rho_{k} a_{k}^{2}}
$$

\section{Numerical Method}

To solve the system of equations (1), first the equation is integrated over the computational cell $\left[x_{i-\frac{1}{2}}, x_{i+\frac{1}{2}}\right]$ to yield the semi-discrete formulation:

$$
\frac{d \mathbf{U}_{i}}{d t}=-\frac{1}{\Delta x}\left(\mathbf{F}_{i+\frac{1}{2}}-\mathbf{F}_{i+\frac{1}{2}}\right)+\mathbf{S}_{i}
$$


Following the standard AUSM method (Liou and Steffen, 1993), the conservative flux vector is split into convective and pressure fluxes:

$$
\mathbf{F}_{i+\frac{1}{2}}=\mathbf{f}_{i+\frac{1}{2}}^{c}+\mathbf{f}_{i+\frac{1}{2}}^{p}
$$

where

$$
\mathbf{f}_{i+\frac{1}{2}}^{c}=\left(\begin{array}{c}
\rho u A \\
\rho u^{2} A \\
\rho u H A \\
0
\end{array}\right)_{i+\frac{1}{2}}, \mathbf{f}_{i+\frac{1}{2}}^{p}=\left(\begin{array}{c}
0 \\
A P \\
0 \\
0
\end{array}\right)_{i+\frac{1}{2}} .
$$

\subsection{Convective flux discretisation}

It is noted that the convective flux can be written explicitly as:

$$
\mathbf{f}^{c}=\dot{m}\left(\begin{array}{c}
1 \\
u \\
H \\
0
\end{array}\right)=\dot{m} \boldsymbol{\Psi}
$$

where $\dot{m}$ is the area-weighted mass flux:

$$
\dot{m}=\rho u A
$$

The numerical flux at cell interface $i+\frac{1}{2}$ is then defined as

$$
\mathbf{f}_{i+\frac{1}{2}}^{c}=\dot{m}^{\star} \boldsymbol{\Psi}^{\star}=\dot{m}^{\star} \frac{1}{2}\left(\boldsymbol{\Psi}_{i}+\mathbf{\Psi}_{i+1}\right)+\frac{1}{2}\left|\dot{m}^{\star}\right|\left(\boldsymbol{\Psi}_{i}-\boldsymbol{\Psi}_{i+1}\right) .
$$

In order to define $\dot{m}^{\star}$ we first define the interface speed of sound $a_{i+\frac{1}{2}}$ and the left and right Mach numbers:

$$
a_{i+\frac{1}{2}}=\frac{\left(a_{i}+a_{i+1}\right)}{2}, \quad M_{i}=\frac{u_{i}}{a_{i+\frac{1}{2}}}, \quad M_{i+1}=\frac{u_{i+1}}{a_{i+\frac{1}{2}}} .
$$


Then we define

$$
\widetilde{M}_{i+\frac{1}{2}}=\mathcal{M}_{4}^{+}\left(M_{i}\right)+\mathcal{M}_{4}^{-}\left(M_{i+1}\right)
$$

where $\mathcal{M}^{+}$and $\mathcal{M}^{-}$are the polynomials introduced by Liou (1996) and the subscript indicates the order of the polynomial used:

$$
\begin{gathered}
\mathcal{M}_{1}^{ \pm}=\frac{1}{2}(M \pm|M|) \\
\mathcal{M}_{2}^{ \pm}= \pm \frac{1}{4}(M \pm 1)^{2} \\
\mathcal{M}_{4}^{ \pm}=\left\{\begin{array}{l}
\mathcal{M}_{1}^{ \pm} / M \\
\pm \mathcal{M}_{1}^{ \pm}\left(1 \mp 16 B \mathcal{M}_{2}^{\mp}\right)
\end{array} \text { where }|M| \geq 1\right.
\end{gathered}
$$

where $B=1 / 8$. Finally, we define:

$$
\dot{m}^{\star}=a_{i+\frac{1}{2}}\left[\frac{(\rho A)_{i}}{2}\left(M_{i+\frac{1}{2}}+\left|M_{i+\frac{1}{2}}\right|\right)+\frac{(\rho A)_{i+1}}{2}\left(M_{i+\frac{1}{2}}-\left|M_{i+\frac{1}{2}}\right|\right)\right] .
$$

At low Mach numbers this approximation approaches a central difference and can suffer from odd-even decoupling (Liou, 1996). In order to suppress this, typically a velocity based dissipation is added to equation (16) (Liou, 2006):

$$
M_{i+\frac{1}{2}}=\widetilde{M}_{i+\frac{1}{2}}-K_{p} \max \left(1-\bar{M}^{2}, 0\right) \frac{P_{i+1}-P_{i}}{\bar{\rho} \bar{a}^{2}}
$$

where $\bar{\rho}$ and $\bar{a}$ represent the arithmetic-averages of the $i$ and $i+1$ values of the respective variables. $K_{p}$ is a constant which is set to unity in this study.

While the above scheme has proved to be remarkably robust, in order to avoid instabilities at discontinuities in the cross-sectional area it is necessary to follow Niu et al. (2008) and replace $\widetilde{M}_{i+\frac{1}{2}}$ in equation (16) with: 


$$
\widetilde{M}_{i+\frac{1}{2}}=\mathcal{M}_{1}^{+}\left(M_{i}\right)+\mathcal{M}_{1}^{-}\left(M_{i+1}\right)
$$

given its greater degree of dissipation at low $M$.

\subsection{Pressure flux discretisation}

The AUSM+ splitting of the non-zero element of the pressure $\mathbf{f}_{i+\frac{1}{2}}^{p}$ flux is defined as:

$$
(A P)_{i+\frac{1}{2}}=A_{i+\frac{1}{2}}\left(\mathcal{P}_{5}^{+}\left(M_{i}\right)(P)_{i}+\mathcal{P}_{5}^{-}\left(M_{i+1}\right)(P)_{i+1}\right)
$$

where the polynomials $\mathcal{P}_{5}^{ \pm}$are given by and the subscripts indicate the order of the polynomial used:

$$
\mathcal{P}_{5}^{ \pm}= \begin{cases}\mathcal{M}_{1}^{ \pm} / M & \text { where }|M| \geq 1 \\ \pm \mathcal{M}_{2}^{ \pm}\left(2 \mp M-16 C M \mathcal{M}_{2}^{\mp}\right) & \text { else. }\end{cases}
$$

with $C=3 / 16$.

In equation (23) $A_{i+\frac{1}{2}}$ is defined separately for both cells next to the interface $i+\frac{1}{2}$, i.e. cell $i$ and $i+1$. In the case of cell $i, A_{i+\frac{1}{2}}$ is assigned its value on the left of the interface, $A_{i+\frac{1}{2}, i}$, while in case of the cell $i+1, A_{i+\frac{1}{2}}$ is set to the value on the right of the interface, $A_{i+\frac{1}{2}, i+1}$. This strategy allows discontinuous variations in the area at the interface $i+\frac{1}{2}$.

Finally, a dissipation term is added to $P_{i+\frac{1}{2}}$. The form of the dissipation term developed by Liou (2006) has been widely used in for both single and two-phase flows (Paillere et al., 2003; Robbins et al., 2013) and is given by:

$$
\begin{aligned}
P_{i+\frac{1}{2}}=\mathcal{P}_{5}^{+}\left(M_{i}\right) P_{i}+\mathcal{P}_{5}^{-}\left(M_{i+1}\right) & P_{i+1} \\
& \quad-K_{u} \mathcal{P}_{(5)}^{+}\left(M_{i}\right) \mathcal{P}_{(5)}^{-}\left(M_{i+1}\right)\left(\rho_{i}+\rho_{i+1}\right) a_{i+\frac{1}{2}}\left(u_{i+1}-u_{i}\right),
\end{aligned}
$$

where $K_{u}$ is constant set to unity. However, in order to maintain stability at discontinuities in the cross-sectional area, equation (25) is altered to incorporate a dependency on the cross-sectional 
area. The corresponding form of the dissipation term applied here is:

$$
\begin{aligned}
P_{i+\frac{1}{2}}=\mathcal{P}_{5}^{+}\left(M_{i}\right) & P_{i}+\mathcal{P}_{5}^{-}\left(M_{i+1}\right) P_{i+1} \\
& -K_{u} \mathcal{P}_{(5)}^{+}\left(M_{i}\right) \mathcal{P}_{(5)}^{-}\left(M_{i+1}\right)\left(\rho_{i}+\rho_{i+1}\right) a_{i+\frac{1}{2}} \frac{\left(A_{i+\frac{1}{2}, i+1} u_{i+1}-A_{i+\frac{1}{2}, i} u_{i}\right)}{\max \left(A_{i+\frac{1}{2}, i+1}, A_{i+\frac{1}{2}, i}\right)} .
\end{aligned}
$$

\subsection{Non-conservative fluxes}

The discretisation of the source term $\mathbf{S}$ containing the non-conservative derivative requires special attention to ensure numerical stability. In particular we take:

$$
\mathbf{S}_{i}=\frac{P_{i}}{\Delta x}\left(\begin{array}{c}
0 \\
\Delta_{i} A \\
0 \\
0
\end{array}\right)
$$

By analogy with the non-conservative terms in the multi-fluid equations studied by Liou et al. (2008), the non zero term in equation (27) is discretised as follows:

$$
\Delta_{i} A=A_{i+\frac{1}{2}, i}-A_{i-\frac{1}{2}, i}
$$

where the areas at the interfaces are taken from within the cell. Analogously, it is easy to show that the non-disturbance relation discussed by Liou et al. (2008) holds here also, i.e. under steady conditions with $u=0$ and $P=$ const this discretisation preserves the relation:

$$
\frac{\partial A P}{\partial x}=P \frac{\partial A}{\partial x}
$$




\subsection{Temporal discretisation}

In this work equation (11) is integrated with respect to time using an $s$-stage second order Low Storage Strong Stability Preserving Runge-Kutta method presented by Ketcheson (2008) denoting the terms on the right-hand side of equation (11) by the operator $\mathcal{L}$ ):

$$
\begin{array}{r}
\mathbf{U}^{0}=\mathbf{U}^{t} \\
\mathbf{U}^{i}=\sum_{j=0}^{i-1}\left(\beta_{i, j} \mathbf{U}^{j}+\Delta t \varepsilon_{i, j} \mathcal{L}\left(t_{n}+c_{k-1} \Delta t, \mathbf{U}^{j}\right)\right) \\
\mathbf{U}^{t+\Delta t}=\mathbf{U}^{s}
\end{array}
$$

for $i=1 \ldots s, s=4$ is used throughout this work and the superscripts $t$ and $t+\Delta t$ indicate the solution at the respective times. $\beta, \varepsilon$ and $c$ are constants, of which the non-zero values are given by (Ketcheson, 2008):

$$
\begin{array}{r}
\beta_{i, i-1}=\left\{\begin{aligned}
\frac{1}{s-1} & 1 \leq i \leq s-1, \\
\frac{1}{s} & i=s,
\end{aligned}\right. \\
\varepsilon_{i, i-1}=\left\{\begin{array}{rr}
1 & 1 \leq i \leq s-1, \\
\frac{s-1}{s} & i=s,
\end{array}\right. \\
\varepsilon_{s, 0}=\frac{1}{s} \\
c_{i}=\frac{i-1}{s-1} .
\end{array}
$$

While the application of this temporal scheme is not required, in practice it has been found that it reduces the likelihood of non-convergence problems in the resolution of real gas Equations of State. 


\section{Numerical Tests}

Since the set of equations (1) is not strictly hyperbolic there is the possibility that for a Riemann problem with two initial states separated by a region in which hyperbolicity does not hold, a nonclassical wave configuration will be produced. In order to assess the ability of the AUSM+-up scheme described above to capture such solutions, the scheme is first applied to two such problems (Tests 1 and 2) for which an exact solution is available for ideal gas flows. This is followed by the application of the scheme to two other tests (Tests 3 and 4). The Test 3 deals with a transient problem presented by Rochette et al. (2012) involving the interaction of a shock wave with a discontinuous reduction in the cross-sectional area. Comparison is made with the solution presented therein. Test 4 investigates the interaction of a rarefaction wave with a discontinuous increase in the cross-sectional area; while there is no reference solution for this problem it represents a physical situation of interest to the authors. It is assumed gas is ideal with an adiabatic index of $\gamma=1.4$, a C.F.L. number of 0.9 is applied and, unless otherwise stated, 1000 computational cells are used.

\subsection{Test 1}

Test 1 is taken from Thanh and Kroner (2012) and involves the interaction between a 1-rarefaction and a resonant surface that induces a further 1-shock in the wave configuration.

Table 1 presents the initial fluid states $(U)$ including fluid density, velocity and pressure on the left $\left(U_{L}\right)$ and right $\left(U_{R}\right)$ of the discontinuity of area, $A$ undergoing a $20 \%$ step enlargment at $x=0$ $\mathrm{m}$. The solution is computed on the interval $[-1,1] \mathrm{m}$.

Figures 1 (a), (b) and (c) show the comparison between the exact solution and the solution using the AUSM+-up scheme for the density, pressure and velocity respectively at $t=0.2 \mathrm{~s}$. As it may be observed, in each case the AUSM+-up scheme accurately captures the solution, with the exception of a slight overshoot in the velocity in the intermediate state following the contact discontinuity at $x=0 \mathrm{~m}$. Importantly, the speed of the additional 1-shock (at $c a . x=0.1 \mathrm{~m}$ ) caused by the interaction with the discontinuous duct area is captured correctly. 


\subsection{Test 2}

This problem was first suggested by Rochette et al. (2012) and was also studied by Thanh and Kroner (2012). It presents a particularly difficult test as the solution contains three co-located stationary waves. Table 2 presents the initial left and right states either side of the discontinuity at $x=0.8 \mathrm{~m}$. The solution is computed on the interval $[0,2] \mathrm{m}$; following Thanh and Kroner (2012) 6000 computational cells are used.

Figures 2 (a) and (b) show the comparison between the exact solution and the AUSM+-up solution for the density and velocity. As a reference, the solution obtained based on the Lax-Friedrichs scheme by Thanh and Kroner (2012) is also presented. As may be observed, the AUSM+-up smears the extrema in both the density and velocity at $x=0.8 \mathrm{~m}$, but captures the remaining features accurately. However its performance is similar to that obtained from Thanh and Kroner (2012), without the need for the solution of the additional non-linear equation employed in the latter case.

\subsection{Test 3}

The following test was also presented by Rochette et al. (2012). It seeks to investigate the interaction of a propagating shock wave with a decrease in the flows cross-sectional area, producing transmitted and reflected shocks. The test involves a shock tube of length $[0,2] \mathrm{m}$ with a discontinuity between the high and low pressure states at $x=0.7 \mathrm{~m}$ while the reduction in cross-sectional area is located $x=1.4 \mathrm{~m}$. Table 3 presents the three initial fluid states.

Figure 3 shows a comparison of Rochette et al.'s (2012) results and those obtained from the AUSM+-up for the density ((a) and (b)) and Mach number ((c) and (d)) at 1.3 and $1.7 \mathrm{~ms}$. As may be observed at $1.3 \mathrm{~ms}$, the AUSM+-up scheme accurately captures the density both in the transmitted and reflected shocks albeit with a slight smearing of the discontinuity at $x=1.4 \mathrm{~m}$, resulting in a slight smearing of the Mach number around this point. Similar trends in the results may be observed at $1.7 \mathrm{~ms}$ with, again, a slight smearing contact discontinuity in the Mach number. 


\subsection{Test 4}

Test 4 is intended to investigate the interaction between a propagating rarefaction wave with a decrease in cross-sectional area. The test involves a shock tube of length $[0,1.5] \mathrm{m}$ with a discontinuity between the high and low pressure states at $x=0.7 \mathrm{~m}$. The reduction in cross-sectional area, $A$ is located at $x=0.5 \mathrm{~m}$. Table 4 shows the three initial fluid states.

Figures 4 (a), (b) and (c) show the fluid density, velocity and pressure profiles respectively at various times. As can be observed in Figure 4 (a), once the rarefaction wave reaches the expansion in shock tube cross-sectional area the density is observed to increase, forming a peak at the discontinuity. Additionally, as seen in previous tests, a stationary wave occurs on the right of this position. No such waves are exhibited in Figure 4 (b) or (c), where discontinuous changes in the velocity and pressure are observed.

As no reference solution is available for this problem and given the behaviour of the scheme at $x=0.5 \mathrm{~m}$, a convergence study is presented. Figures 5 (a), (b) and (c) show the solutions obtained at $0.4 \mathrm{~ms}$ using 100, 500, 1000 and 10000 cells respectively. It is clear from the figures that convergence is obtained. In particular, the magnitude of the density peak at $x=0.5 \mathrm{~m}$ is independent of the discretisation used. This can more clearly be observed in Figure 6 showing the corresponding variation in density. As may be seen, all three discretisation levels produce the same peak density.

\section{Two-phase tests}

Having established the capability of the AUSM+-up scheme presented above in handling ideal gas flow through discontinuous area ducts, we now move on to its application to two-phase mixtures based on the HEM assumption. Two test cases (Tests 5 and 6) using a liquid-vapour $\mathrm{CO}_{2}$ as the working fluid are dealt with. The first (Test 5) is a shock tube problem similar to Test 1 (Section 4). A convergence study is performed and the impact of vapour volume fraction, $\alpha_{v}$ on the predictions is investigated. The remaining test investigates the dynamic interaction of a discontinuous change in the cross-sectional area with a shock (Test 6). In each case the problem is solved on the interval $[0,1.5] \mathrm{m}$ with a 1000 computational cells, a C.F.L. number of 0.3 and the PR EoS are applied. 


\subsection{Test 5}

Table 5 presents the fluid states to the left and right of the discontinuity at $x=0.6 \mathrm{~m}$ for Test 5 . For the sake of analysis, discontinuities in pressure and cross-sectional area as well as the initial vapour volume fraction $\left(\alpha_{v}\right)$ are introduced.

Figures 7 (a), (b), (c), (d) and (e) show the corresponding density, velocity, $\alpha_{v}$, Mach number and entropy, respectively, at various times. As may be observed, upon initiation of the problem a shock propagates to the right and is accompanied by an evaporation jump (such behaviour is typical of two-phase shock tube problems (Faucher et al., 2000)). This is followed by a slower moving contact discontinuity. A clear peak can be seen at $x=0.6 \mathrm{~m}$, with an increase in the density to left of it which has a marginal effect on the vapour volume fraction. The magnitude of this peak does not increase with time, and was found to be mesh independent. The velocity jump at $x=0.6 \mathrm{~m}$ (Figure 7 (b)) is similar to that observed previously (see Figure 1). Also, as observed in 7 (e) the entropy increases with the progression of the contact discontinuity, as expected.

As no reference solution for this problem exists, as in the case of Test 4, a convergence study is presented in Figure 8 (a), (b), (c) and (d) showing the solutions obtained at 0.4 ms using 100, 500, 1000 and 10000 cells respectively. It is clear from the figures that convergence is achieved.

Figures 9 shows the corresponding data as in Figure 7 for various initial $\alpha_{v}$ on the right of the discontinuity. As may be observed, the speed of the right-going shock wave is markedly affected by the initial $\alpha_{v}$ in this region. This is to be expected given the non-linearity of equation (10). Interestingly, the increase in $\alpha_{v}$ observed for $\alpha_{v}=0.9$ to the left of the shock wave becomes smaller for $\alpha_{v}=0.7$ and a decrease is seen in the case of $\alpha_{v}=0.5$. This behaviour is a result of the the curvature of the $\mathrm{CO}_{2}$ isentropes within the two-phase region.

\subsection{Test 6}

Test 6 seeks to investigate the interaction of a shock wave with a restriction in the cross-sectional area of the shock tube. The test involves a discontinuity between the high and low pressure states at $x=0.6 \mathrm{~m}$. The reduction in cross-sectional area, $A$, is located $x=0.9 \mathrm{~m}$. Table 6 presents the three initial states.

Figures 10 (a), (b), (c) and (d) show the fluid density, velocity, $\alpha_{v}$ and Mach number respectively 
at various times. As may be observed, at $2 \mathrm{~ms}$ the shock produced has reached the restriction and, as in Test 3, has propagated into the restriction. A reflected wave is observed resulting in a decrease in the velocity (Figure 10 (b)); also, the reflected shock results in a slight increase in the density, which is clear at $4 \mathrm{~ms}$ (Figure 10 (a)), and is accompanied by a relatively small decrease in the vapour quality.

\section{Conclusion}

In this study, the AUSM+-up scheme was extended for the simulation of two-phase or flashing flows in ducts with discontinuous or rapidly changing cross-sectional areas. Such information is of vital relevance in many engineering applications including the design of refrigeration systems, cavitation in automotive fuel injection systems, high pressure pipeline transportation, and liquid evaporation during loss-of-coolant accidents in nuclear reactors.

The scheme, drawing on work from multi-fluid flows, allows a simple discretisation of the nonconservative terms in the governing equations. An additional scaling of the pressure-based dissipation is added in order to ensure stability across the cross-sectional area change. Importantly, and in contrast to other methods, no equation of state is assumed and thus the extension to two-phase flows is straightforward.

The scheme is first verified using two test Riemann problems for an ideal gas, both of which contained resonance in the solution. In the first of the two cases, it is found that the full wave solution is successfully computed. In the second case, where several waves coexist at a single point, while there is smearing of the front of the rarefaction, remainder of the solution is accurate and the resolution at this point is close to that obtained by Thanh and Kroner (2012).

A transient verification test involving the dynamic interaction of a shock with a restriction showed that the scheme computed both the reflected and transmitted shocks correctly. The application of the scheme to another transient problem involving the interaction between a rarefaction wave and an expansion demonstrated the formation of a standing wave.

Investigations using the scheme for a gas-liquid mixture (saturated two-phase $\mathrm{CO}_{2}$ ) in a shock tube test incorporating a rapid change in the cross-sectional area showed that it is sufficiently robust to resolve this more complex problem. A further test using the $\mathrm{CO}_{2}$ mixture was performed 
to simulate the interaction of a shock wave with a discontinuous change in cross-sectional area. In summary, the scheme developed in this work has been shown to be sufficiently robust for capturing the dynamics of the flashing flow in ducts with rapidly changing cross-sectional area. Current work by the authors includes the extension of the scheme to enable the capture of non-equilibrium effects during the phase transition process described using a homogeneous relaxation or two-fluid model.

\section{Acknowledgements}

This work was supported by the UK Engineering and Physical Science Research Council (EPSRC reference EP/K000446/1) and the European Union 7th Framework Programme FP7-ENERGY2012-1-2STAGE under grant agreement number 309102.

\section{References}

Abgrall, R., Saurel, R., 2003. Discrete equations for physical and numerical compressible multiphase mixtures. Journal of Computational Physics 186 (2), 361-396.

Andrianov, N., Warnecke, G., 2004. On the solution to the Riemann problem for the compressible duct flow. SIAM Journal on Applied Mathematics 64 (3), 878-901.

Blinkov, V. N., Jones, O. C., Nigmatulin, B. I., 1993. Nucleation and flashing in nozzles-2. Comparison with experiments using a five-equation model for vapor void development. International Journal of Multiphase Flow 19 (6), 965-986.

Bouchut, F., 2004. Nonlinear Stability of Finite Volume Methods for Hyperbolic Conservation Laws: And WellBalanced Schemes for Sources. Frontiers in Mathematics. Springer Verlag, NY.

Clain, S., Rochette, D., 2009. First- and second-order finite volume methods for the one-dimensional nonconservative Euler system. Journal of Computational Physics 228 (22), 8214-8248.

Cortes, J., 2002. On the construction of upwind schemes for non-equilibrium transient two-phase flows. Computers \& Fluids 31 (2), 159-182.

Edwards, J., Franklin, R., Liou, M., 2000. Low-diffusion flux-splitting methods for real fluid flows with phase transitions. AIAA journal 38 (9).

Ettouney, R., El-Rifai, M., Elzoubier, A., 2012. Emergency venting into redundant pipelines. Journal of Loss Prevention in the Process Industries 25 (4), 739-745.

Faucher, E., Hérard, J.-m., Barret, M., Toulemonde, C., 2000. Computation of flashing flows in variable cross-section ducts. International Journal of Computational Fluid Dynamics 13, 365-391. 
Goncalvès, E., Patella, R. F., 2009. Numerical simulation of cavitating flows with homogeneous models. Computers \& Fluids 38 (9), 1682-1696.

Goncalvès, E., Patella, R. F., 2010. Numerical study of cavitating flows with thermodynamic effect. Computers \& Fluids 39 (1), 99-113.

Hammer, M., Ervik, A., Munkejord, S., 2013. Method using a density-energy state function with a reference equation of state for fluid-dynamics simulation of vapor-liquid-solid carbon dioxide. Industrial \& Engineering Chemistry Research 52 (29), 9965-9978.

Han, E., Hantke, M., Warnecke, G., 2012. Exact riemann solutions to compressible euler equations in ducts with discontinuous cross-section. Journal of Hyperbolic Differential Equations 9 (3), 403-449.

Helluy, P., Hérard, J.-M., Mathis, H., 2012. A well-balanced approximate Riemann solver for compressible flows in variable cross-section ducts. Journal of Computational and Applied Mathematics 236 (7), 1976-1992.

Ishii, M., Hibiki, T., 2006. Thermo-fluid Dynamics of Two-Phase Flow. Smart Energy Systems. Springer, NY.

Ketcheson, D. I., 2008. Highly efficient strong stability preserving Runge-Kutta methods with low-storage implementations. SIAM Journal of Scientific Computing 30 (4), 2113-2136.

Kröner, D., Thanh, M. D., 2006. Numerical solutions to compressible flows in a nozzle with variable cross-section. SIAM Journal on Numerical Analysis 43 (2), 796-824.

Liou, M., 1996. A Sequel to AUSM: AUSM+. Journal of Computational Physics 129 (2), 364-382.

Liou, M., 2006. A sequel to AUSM, Part II: AUSM+-up for all speeds. Journal of Computational Physics 214 (1), $137-170$.

Liou, M., Chang, C., Nguyen, L., Theofanous, T. G., 2008. How to solve compressible multifluid equations: A simple, robust, and accurate method. AIAA 46 (9), 2345-2356.

Liou, M., Steffen, C., 1993. A new flux splitting scheme. Journal of Computational Physics 107, 23-39.

Martynov, S. B., Mason, D. J., Heikal, M. R., 2006. Numerical Simulation of Cavitation Flows Based on Their Hydrodynamic Similarity. International Journal of Engine Research 7 (3), 283-296.

Nigmatulin, R., Soplenkov, K., 1994. Non-stationary outflow and rarefaction waves in flashing liquid. Nuclear Engineering and Design 151, 131-144.

Niu, Y.-Y., Lin, Y.-C., Chang, C.-H., 2008. A further work on multi-phase two-fluid approach for compressible multiphase flows. International Journal for Numerical Methods in Fluids 58 (8), 879-896.

Paillere, H., Corre, C., Garcia Cascales, J., 2003. On the extension of the AUSM+ scheme to compressible two-fluid models. Computers \& Fluids 32 (6), 891-916.

Peng, D.-Y., Robinson, D. B., 1976. A New Two-Constant Equation of State. Industrial \& Engineering Chemistry Fundamentals 15 (1), 59-64.

Picard, D., Bishnoi, P., 1987. Calculation of the thermodynamic sound velocity in two-phase multicomponent fluids. International Journal of Multiphase Flow 13 (3), 295-308. 
Poling, B., Prausnitz, J., O’Connell, J., 2001. The properties of gases and liquids. McGraw-Hill.

Robbins, D., Cant, R., Gladden, L., 2013. Development of accurate, robust liquid equations of state for multi-phase CFD simulations with a modified AUSM+-up scheme. Computers \& Fluids 77, 166-180.

Rochette, D., Clain, D., Buffard, T., 2005. Numerical scheme to complete a gas flow in variable porosity media. International Journal of Computational Fluid Dynamics 19 (4), 299-309.

Rochette, D., Clain, S., Bussiere, W., 2012. Unsteady compressible flow in ducts with carying cross-section: comparison between the non conservative euler system and the axisymmetric flow model. Computers \& Fluids 53, $53-78$.

Saurel, R., Petitpas, F., Abgrall, R., 2008. Modelling phase transition in metastable liquids: application to cavitating and flashing flows. Journal of Fluid Mechanics 607, 313-350.

Simões-Moreira, J. R., Bullard, C. W., 2003. Pressure drop and flashing mechanisms in refrigerant expansion devices. International Journal of Refrigeration 26 (7), 840-848.

Stewart, H. B., Wendroff, B., 1984. Two-phase flow: Models and methods. Journal of Computational Physics 56 (3), $363-409$.

Thanh, M., Kroner, D., 2012. Numerical treatment of nonconservative terms in resonant regime for fluid flows in a nozzle with variable cross-section. Computers \& Fluids 66, 130-139.

Yeom, G.-S., Chang, K.-S., 2013. A modified HLLC-type riemann solver for the compressible six-equation two-fluid model. Computers \& Fluids 76, 86-104. 
Table 1: Initial states for Test 1.

\begin{tabular}{ccccc}
\hline & $\rho\left(\mathrm{kg} \mathrm{m}^{-3}\right)$ & $u\left(\mathrm{~m} \mathrm{~s}^{-1}\right)$ & $P(\mathrm{~Pa})$ & $A$ \\
\hline$U_{L}$ & 5 & 0.5 & 8 & 1.0 \\
$U_{R}$ & 1 & 0.8 & 1 & 1.2 \\
\hline
\end{tabular}

Table 2: Initial states for Test 2.

\begin{tabular}{ccccc}
\hline & $\rho\left(\mathrm{kg} \mathrm{m}^{-3}\right)$ & $u\left(\mathrm{~m} \mathrm{~s}^{-1}\right)$ & $P(\mathrm{~Pa})$ & $A$ \\
\hline$U_{L}$ & 1.3 & 2 & 1 & 1 \\
$U_{R}$ & 2.363115 & 3.675948 & 1 & 0.7 \\
\hline
\end{tabular}

Table 3: Initial states for Test 3.

\begin{tabular}{ccccc}
\hline & $\rho\left(\mathrm{kg} \mathrm{m}^{-3}\right)$ & $u\left(\mathrm{~m} \mathrm{~s}^{-1}\right)$ & $P(\mathrm{MPa})$ & $A$ \\
\hline$U_{1}$ & 35.6 & 0 & 3 & 1 \\
$U_{2}$ & 1.1867 & 0 & 0.1 & 1 \\
$U_{3}$ & 1.1867 & 0 & 0.1 & 0.4 \\
\hline
\end{tabular}

Table 4: Initial states for Test 4.

\begin{tabular}{ccccc}
\hline & $\rho\left(\mathrm{kg} \mathrm{m}^{-3}\right)$ & $u\left(\mathrm{~m} \mathrm{~s}^{-1}\right)$ & $P(\mathrm{MPa})$ & $A$ \\
\hline$U_{1}$ & 35.6 & 0 & 3 & 1 \\
$U_{2}$ & 35.6 & 0 & 3 & 0.4 \\
$U_{3}$ & 1.1867 & 0 & 0.1 & 0.4 \\
\hline
\end{tabular}

Table 5: Initial states for Test 5.

\begin{tabular}{cccccc}
\hline & $\rho\left(\mathrm{kg} \mathrm{m}^{-3}\right)$ & $u\left(\mathrm{~m} \mathrm{~s}^{-1}\right)$ & $P(\mathrm{MPa})$ & $A$ & $\alpha_{v}$ \\
\hline$U_{1}$ & 829.1 & 0 & 5 & 1 & 0 \\
$U_{2}$ & 126.8 & 0 & 4 & 0.5 & 0.9 \\
\hline
\end{tabular}

Table 6: Initial states for Test 6.

\begin{tabular}{cccccc}
\hline & $\rho\left(\mathrm{kg} \mathrm{m}^{-3}\right)$ & $u\left(\mathrm{~m} \mathrm{~s}^{-1}\right)$ & $P(\mathrm{MPa})$ & $A$ & $\alpha_{v}$ \\
\hline$U_{1}$ & 829.1 & 0 & 5 & 1 & 0 \\
$U_{2}$ & 126.8 & 0 & 4 & 1 & 0.9 \\
$U_{3}$ & 126.8 & 0 & 4 & 0.5 & 0.9 \\
\hline
\end{tabular}




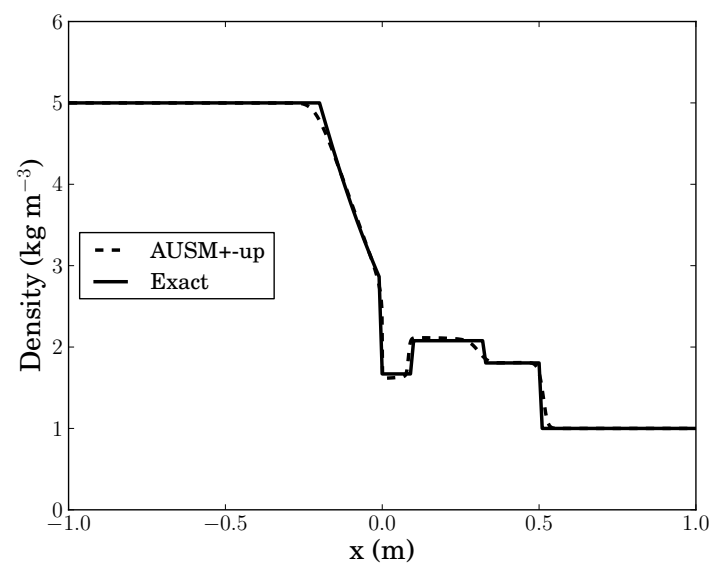

(a)

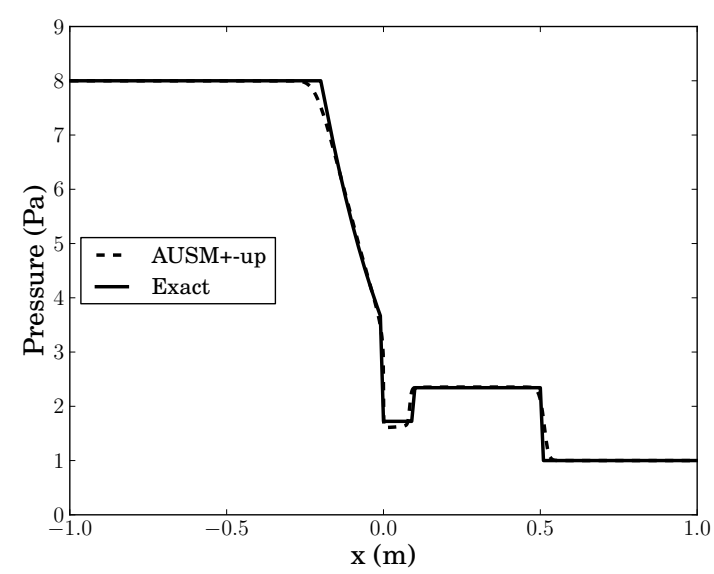

(b)

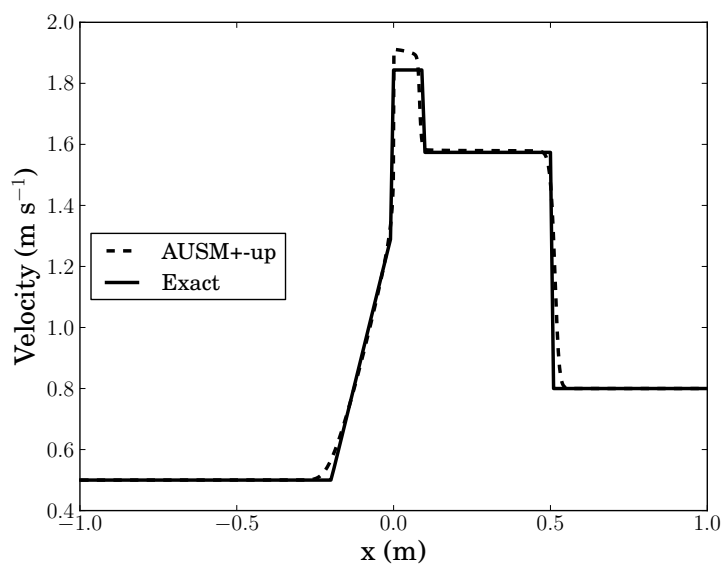

(c)

Figure 1: Profiles of the density (a), pressure (b) and velocity (c) at at $t=0.2 \mathrm{~s}$ for Test 1. 


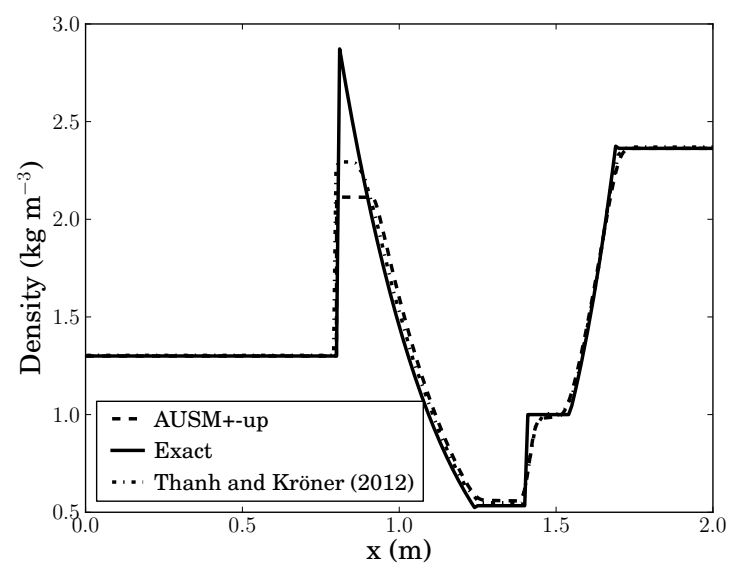

(a)

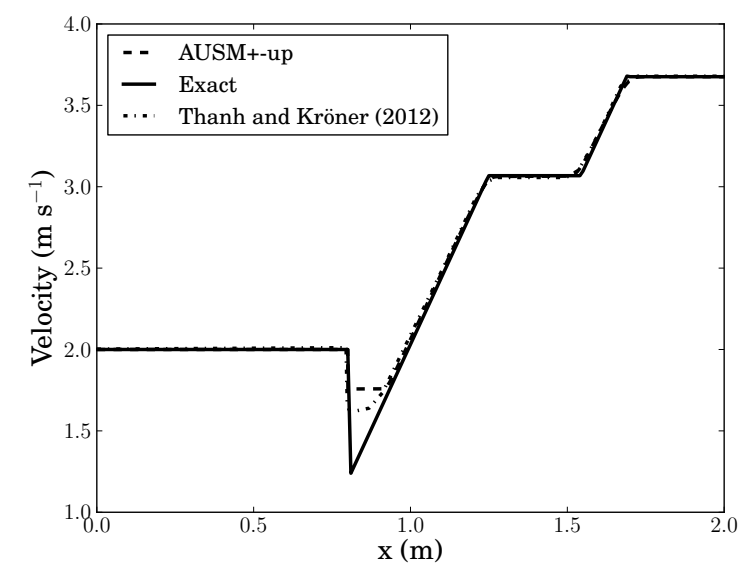

(b)

Figure 2: Profiles of the density (a) and velocity (b) at at $t=0.2 \mathrm{~s}$ for Test 2. 


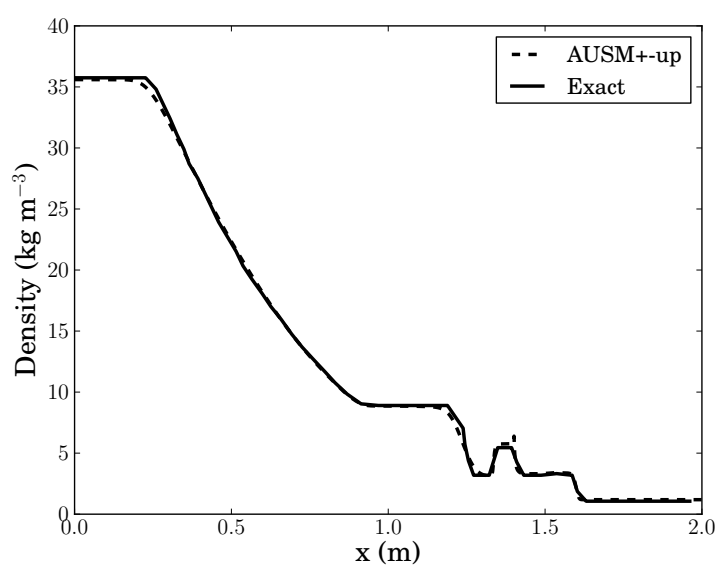

(a)

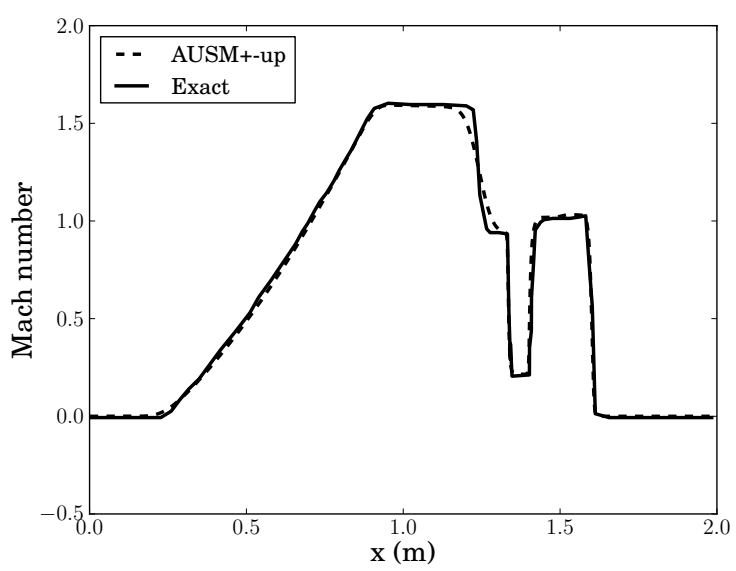

(c)

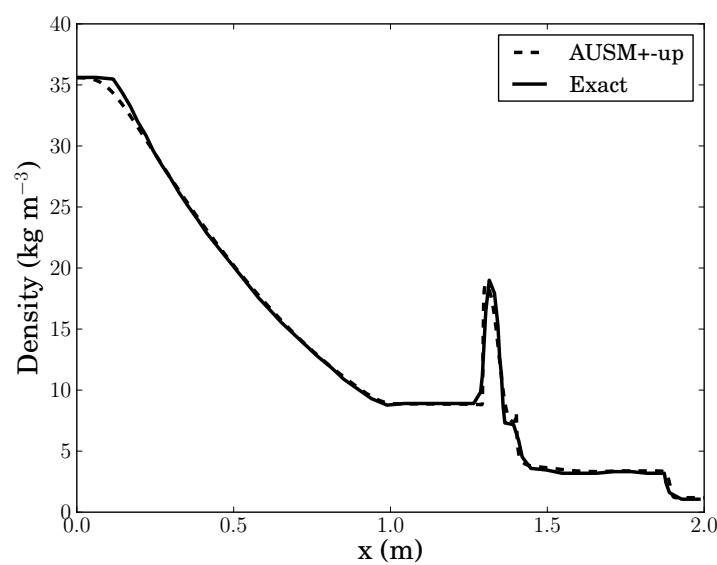

(b)

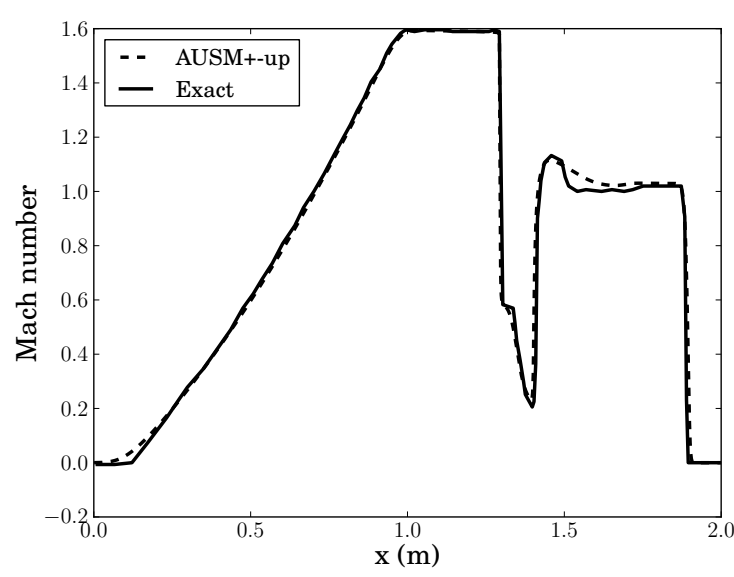

(d)

Figure 3: Profiles of the density ((a) and (b)) and Mach number and ((c) and (d)) at $t=1.3$ and $t=1.7 \mathrm{~ms}$ respectively for Test 3 . 


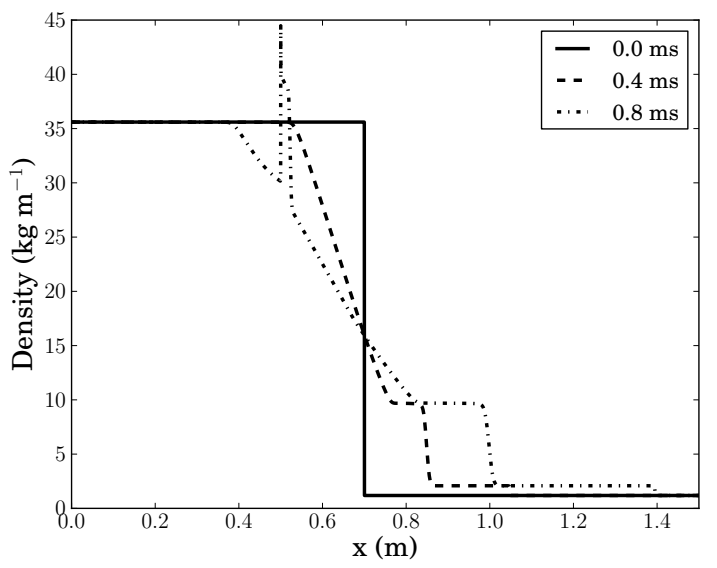

(a)

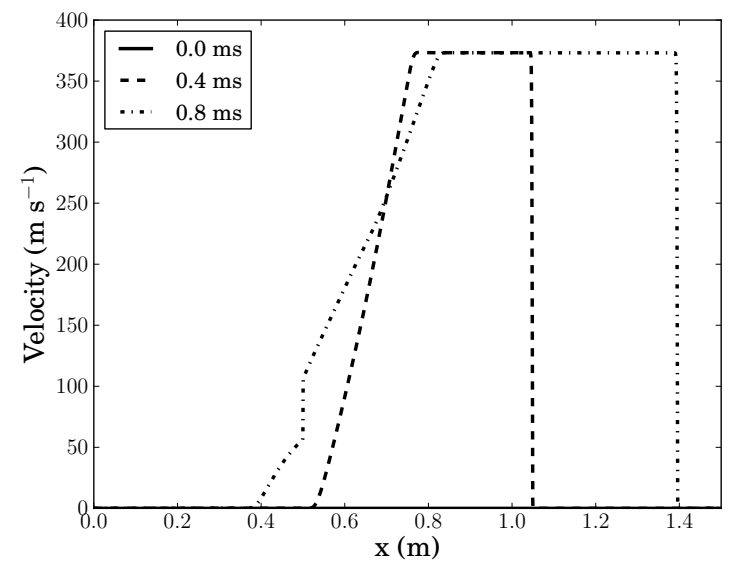

(b)

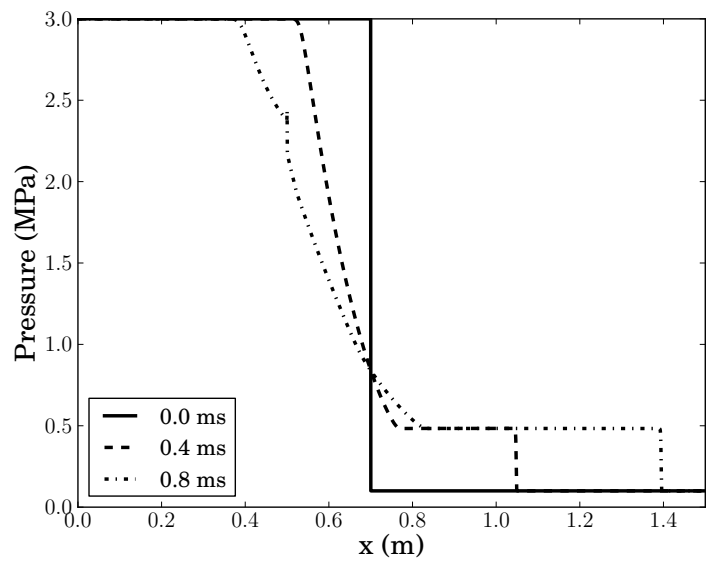

(c)

Figure 4: Profiles of the density (a), velocity (b) and (c) resp. for Test 4. 


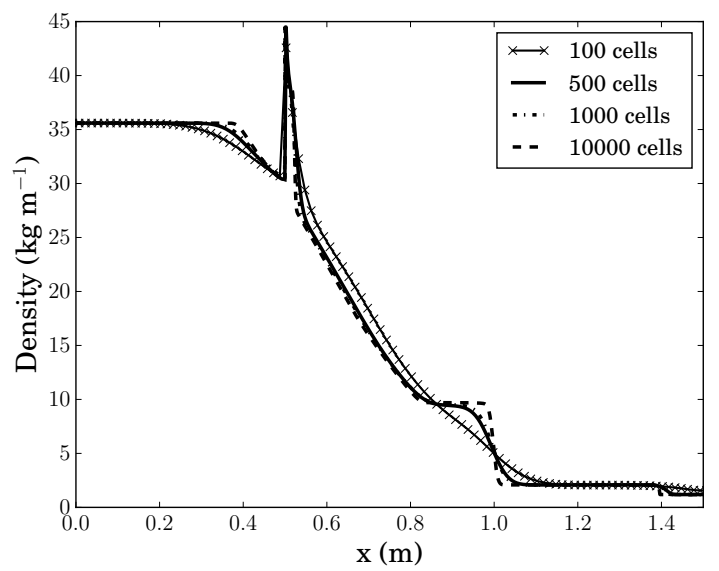

(a)

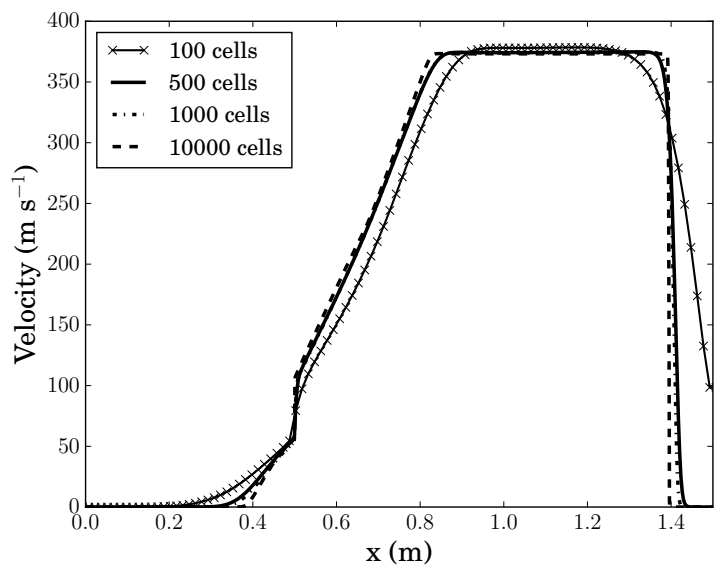

(b)

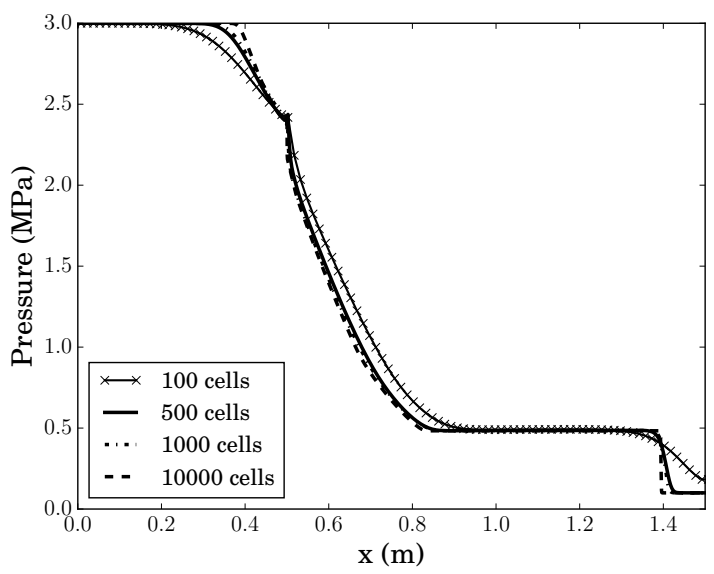

(c)

Figure 5: Profiles of the density (a), velocity (b) and (c) obtained using various mesh sizes for Test 4. 


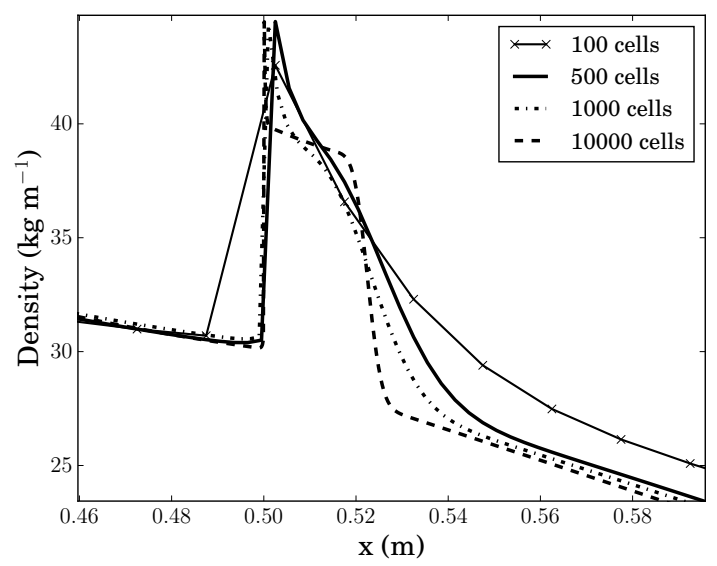

Figure 6: Close up of the standing wave at cross-sectional discontinuity. 


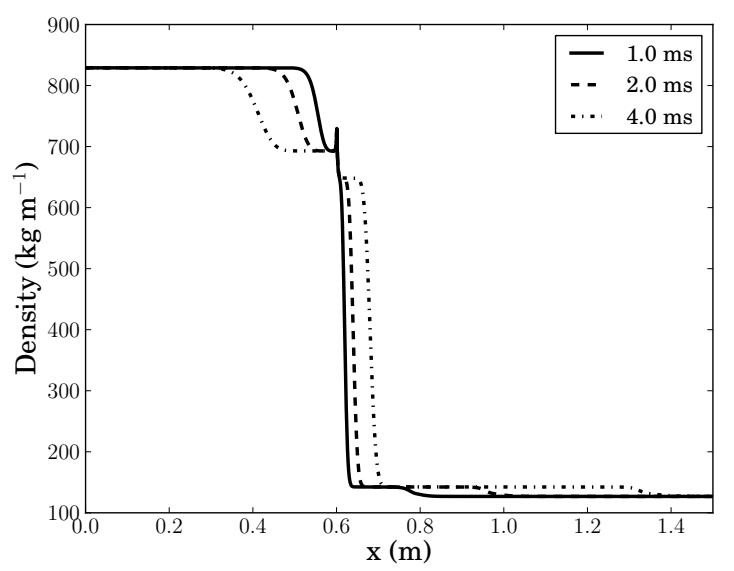

(a)

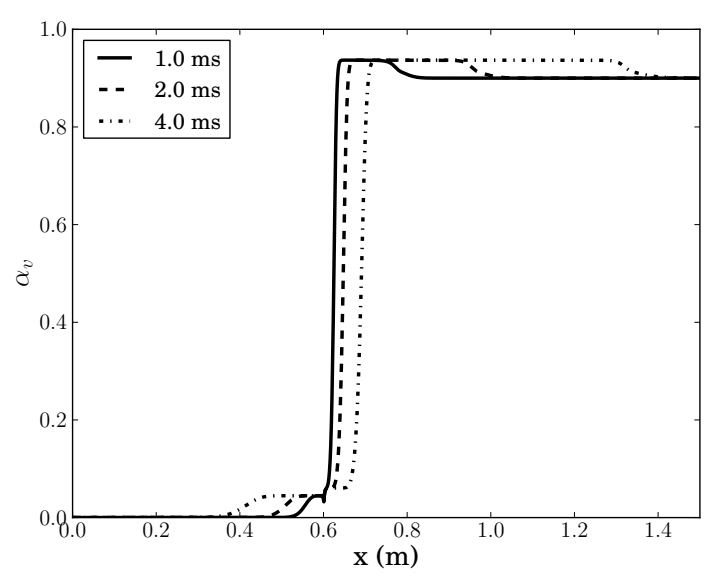

(c)

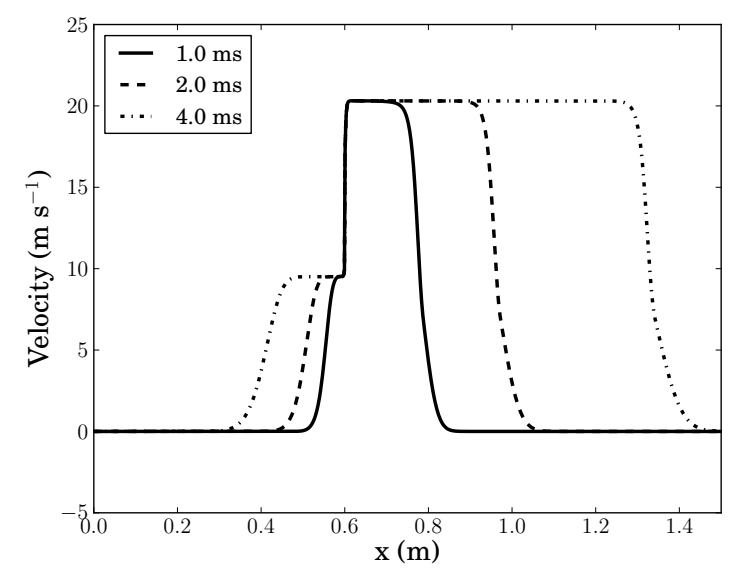

(b)

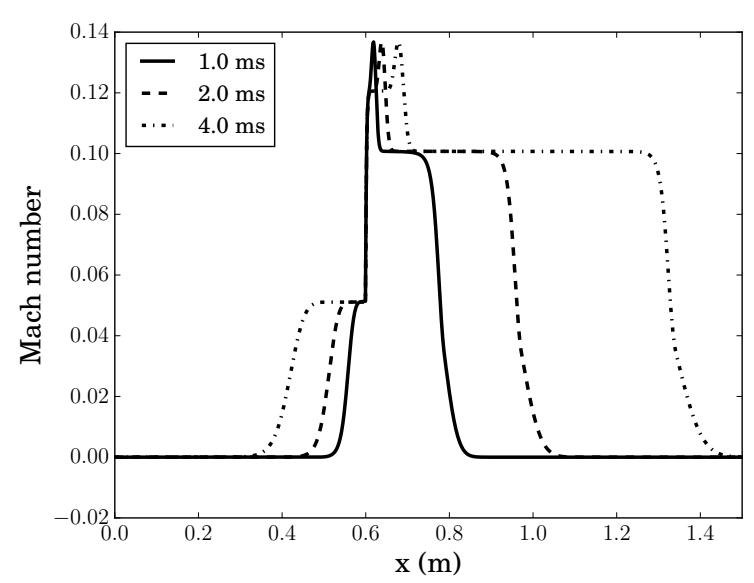

(d)

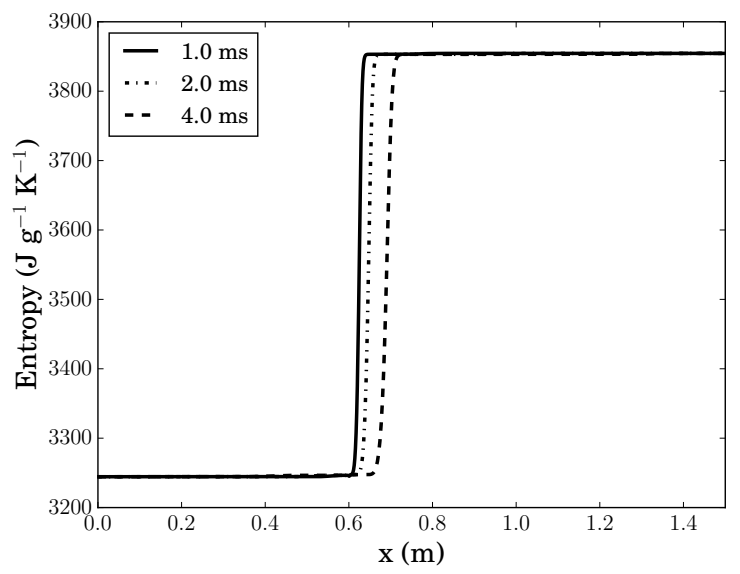

(e)

Figure 7: Profiles of density (a), velocity (b), vapour fraction (c), Mach number (d) and entropy at $t=0,2$ and 4 ms respectively for Test 5 . 


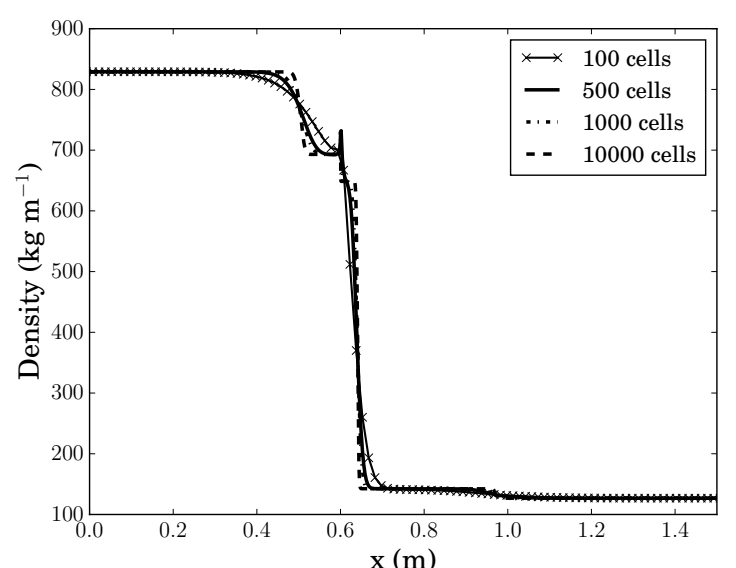

(a)

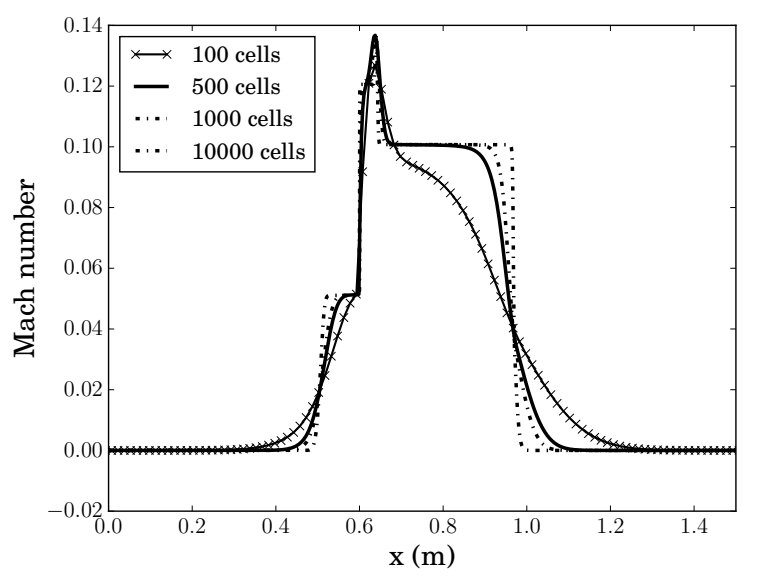

(c)

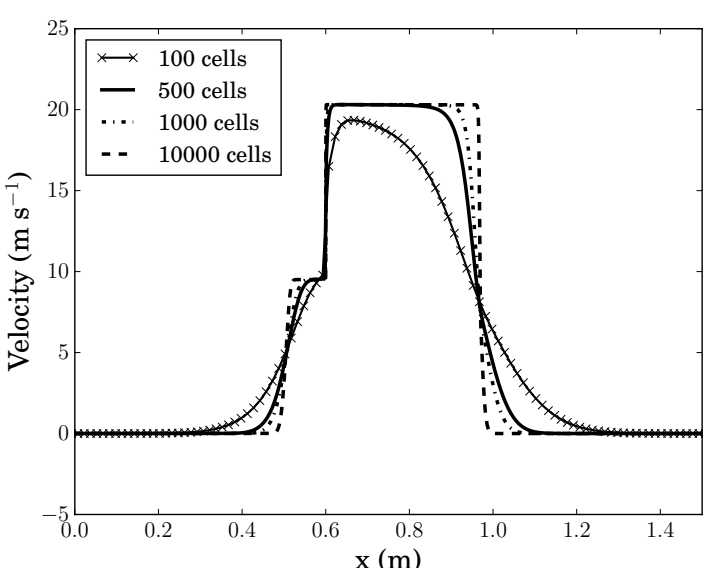

(b)

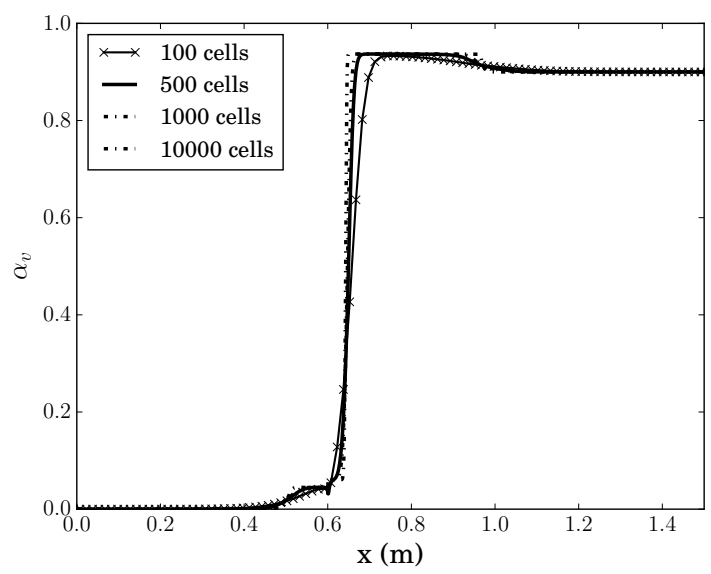

(d)

Figure 8: Profiles of density (a), velocity (b), vapour fraction (c) and Mach number (d) obtained using various mesh sizes for Test 5 . 


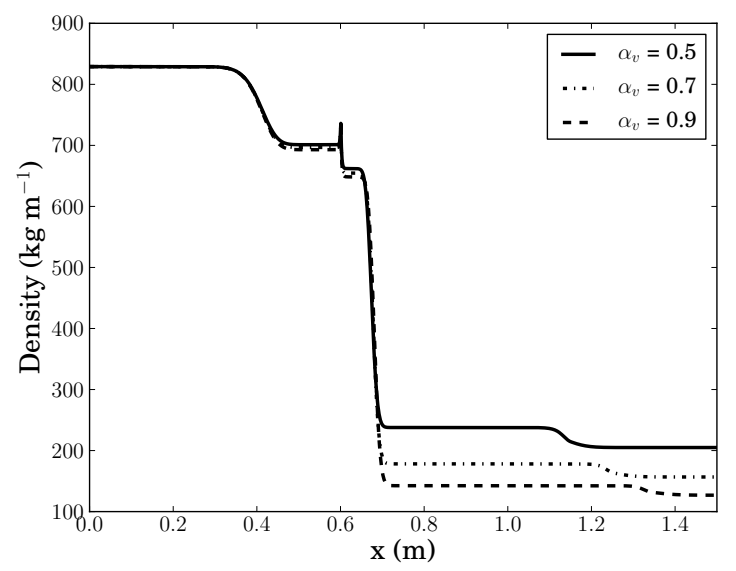

(a)

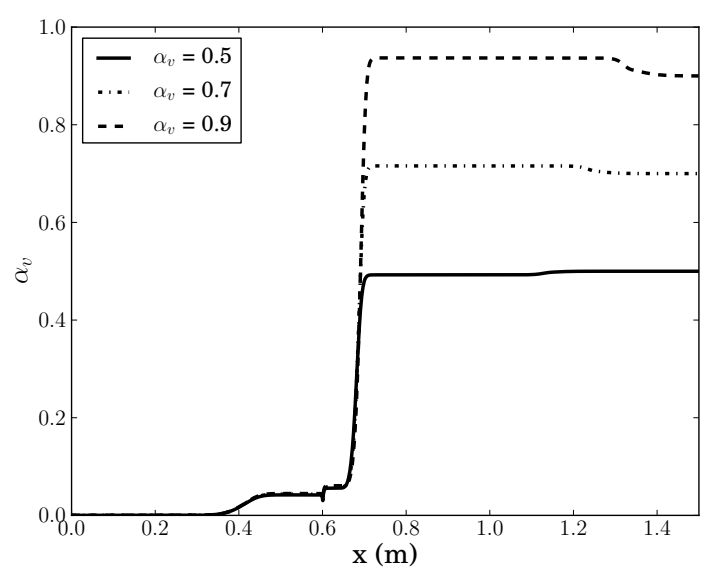

(c)

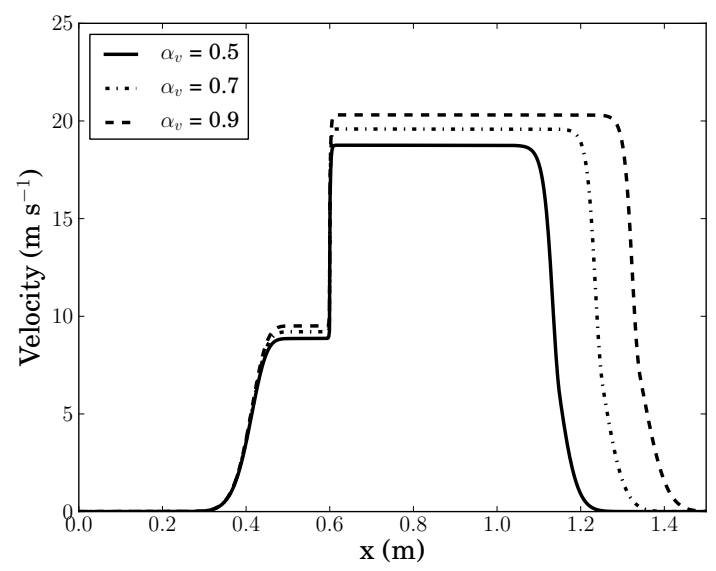

(b)

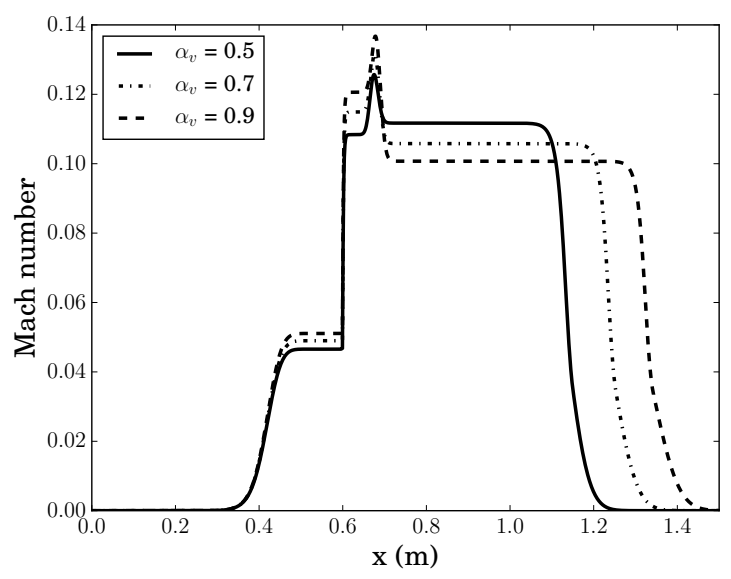

(d)

Figure 9: Profiles of density (a), velocity (b), vapour fraction (c) and Mach number (d) at 4 ms respectively for Test 5 with varying initial vapour volume fractions. 


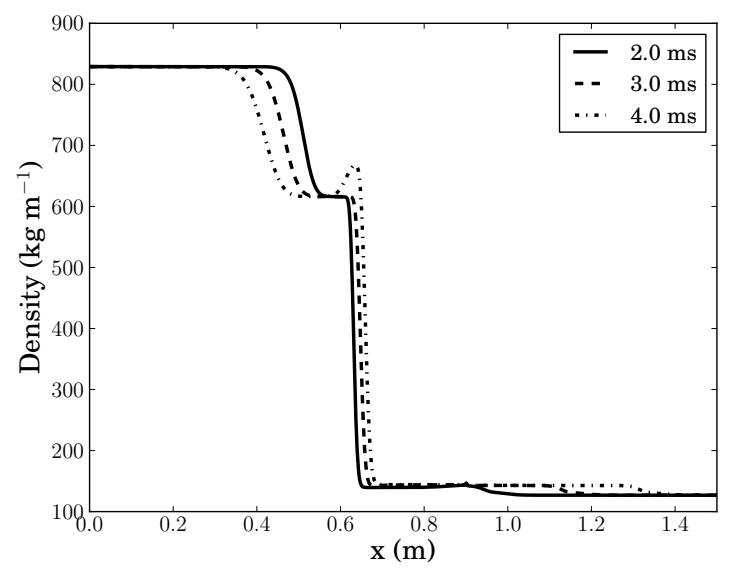

(a)

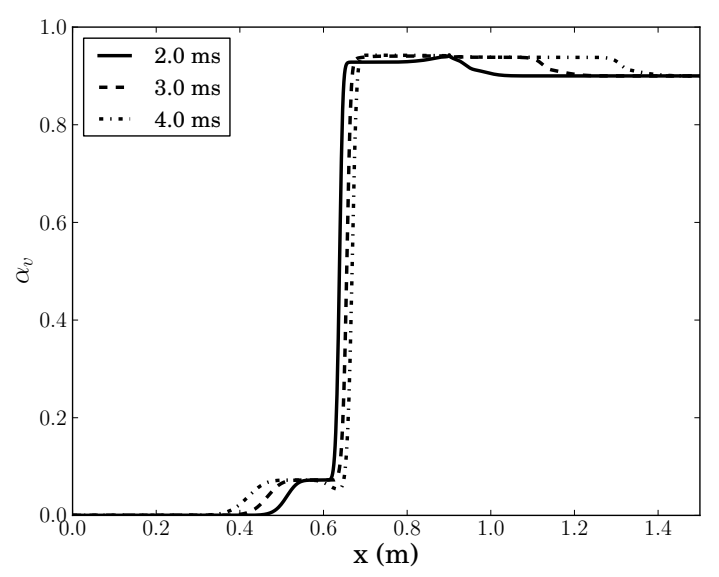

(c)

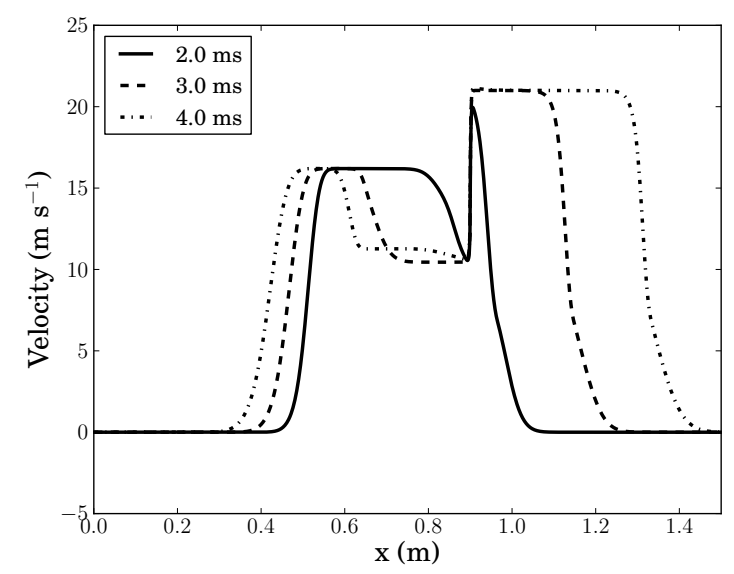

(b)

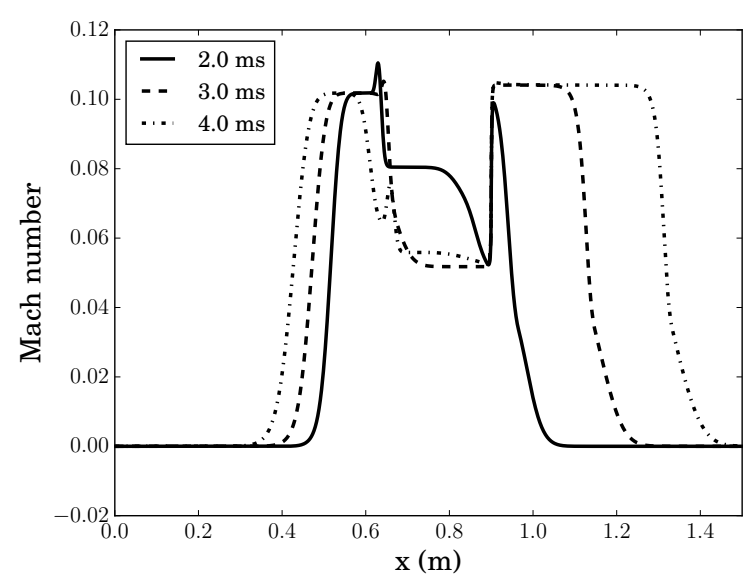

(d)

Figure 10: Profiles of density (a), velocity (b), vapour fraction (c) and Mach number (d) at $t=2,3$ and $4 \mathrm{~ms}$ respectively for Test 6 . 